\title{
Correction to: Symptoms Suggestive of Dysphagia and the Quality of Life in Cocaine and/or Crack Users
}

\author{
Karini Mayer Silva da Cunha ${ }^{1}$ (i) - Taís de Campos Moreira ${ }^{2}$. Sheila Tamanini de Almeida ${ }^{3}$. \\ Helena Maria Tannhauser Barros ${ }^{4} \cdot$ Maristela Ferigolo $^{5}$
}

Published online: 6 September 2019

(c) Springer Science+Business Media, LLC, part of Springer Nature 2019

\section{Correction to: Dysphagia \\ https://doi.org/10.1007/s00455-019-10013-0}

The original version of this article unfortunately contained a mistake. The spelling of the Sheila Taminini de Almeida name was incorrect.

The correct name should be Sheila Tamanini de Almeida.

The original article has been corrected.

The original article can be found online at https://doi.org/10.1007/ s00455-019-10013-0.

Karini Mayer Silva da Cunha

karinicunha@gmail.com

1 Associação Mario Tannhauser de Ensino, Pesquisa e Assistência, R. Sarmento Leite, 246/601, Bom Fim, Porto Alegre, RS 90030-030, Brazil

2 R. Irmão José Otão, 575/602, Bom Fim, Porto Alegre, RS 90020-090, Brazil

3 Departamento de Fonoaudiologia, Fundação Universidade Federal de Ciências da Saúde de Porto Alegre, Rua Sarmento Leite, 245, Centro, Porto Alegre, RS 90050-170, Brazil

4 Departamento de Ciências Fisiológicas, Fundação Universidade Federal de Ciências da Saúde de Porto Alegre, Rua Sarmento Leite, 245, Centro, Porto Alegre, RS 90050-170, Brazil

5 Departamento de Farmacologia e Toxicologia, Farmacologia, Fundação Universidade Federal de Ciências da Saúde de Porto Alegre, Rua Sarmento Leite, 245, Centro, Porto Alegre, RS 90050-170, Brazil 